\title{
A Social Media Platform in Higher Education
}

\author{
Evgeny Bogdanov, Freddy Limpens, Na Li, Sandy El Helou, Christophe Salzmann, Denis Gillet \\ School of Engineering \\ Ecole Polytechnique Fédérale de Lausanne \\ Lausanne, Switzerland \\ \{evgeny.bogdanov,freddy.limpens,na.li,sandy.elhelou,christophe.salzmann,denis.gillet\}@epfl.ch
}

\begin{abstract}
This paper reports on the successful use of Graasp, a social media platform, by university students for their collaborative work. Graasp features a number of innovations, such as administrator-free creation of collaborative spaces, a context-aware recommendation and privacy management. In the context of a EU-funded project involving large test beds, we have been able to extend this platform with lightweight tools (widgets) aimed for learning and competence development and to validate its usefulness in a collaborative learning context.
\end{abstract}

Keywords-collaborative learning; social media; competence development; education; context-awareness; widgets; opensocial

\section{INTRODUCTION}

Regarding collaborative tools for learning, we observe today a convergence of social web platforms and learning tools, with social media containers such as iGoogle being used for learning purposes and typical Learning Management Systems (LMS) such as moodle.org introducing widgets and dashboard features similarly to iGoogle. Learners are looking for more flexibility and compatibility with the social Web tools they now use daily [1] while also being more and more keen on keeping an eye on privacy and sharing settings. Graasp ${ }^{1}$ (formerly Graaasp) is a collaborative Web-based platform that combines flexibility of a social media container with learning applications. In this regard, it particularly suits the self-directed learning paradigm whereby learners progress in a given discipline through personal and collaborative projects lead with a greater degree of autonomy [2]. This does not mean that teachers are no longer required, on the contrary. However, it is needless to say that collaborative projects-based learning activities are taking a greater part in students' work, especially in engineering studies, and that new tools are, therefore, needed to help students cope with this even more demanding task. This is why self-directed learning is at the center of the ROLE ${ }^{2}$ (Responsive Open Learning Environment) EU-funded project in which Graasp is not only tested and validated, but also extended following a participatory design approach. Moreover, this paper reports on our partnership with the SWITCH PLE project, hosted by a network of Swiss universities that are willing to complement their institutional learning tools with Graasp.

The paper is organized as follows. Section II introduces the challenges and the requirements. In Section III we reveal the Graasp's main features and discuss how these features satisfy

\footnotetext{
${ }^{1} \mathrm{http} / /$ graasp.epfl.ch

${ }^{2} \mathrm{http}: / /$ www.role-project.eu
}

the requirements of higher education. Section IV presents the preliminary Graasp's evaluation and explains its deployment setting in educational environment. Section V summarizes the paper and provides an overview of future plans.

\section{Problem STATEMENT AND BACKGROUND}

\section{A. Graasp as a Personal Learning Environment}

The aim of this paper is to show that social media platforms in general, and Graasp in particular, fill in a gap within the offer of learning tools in an academic context. In this respect Graasp is meant to serve as a Personal Learning Environment (PLE). A PLE can be defined as a set of tools centered on learners and designed to help them manage autonomously their interactions with people and resources relevant to their learning context $[3,4]$. To give a non-exhaustive example, a typical PLE could be composed of a mail client (e.g., Thunderbird), an account in a professional social networking platform (e.g., LinkedIn), an account at delicious.com and the corresponding Firefox plugin to manage Web bookmarks, and an access to a university course material catalogue. While not aimed at replacing these tools or other Web 2.0 and other user-centered tools, Graasp provides in one online platform the same functionalities. It can be used for communicating with peers or collecting and organizing resources coming either from the Web or from any third party repository. Moreover, it allows learners to deal with different contexts with the notion of a space, which is meant to help users organize their online activities, resources, contacts, etc. (see Section III.A for a detailed description of the space notion)

One important question is how can PLEs enhance current LMSs and learning tools in general. Henry et al. in [5] summarize the ways PLEs could intervene in the field of learning. In self-learning and reflexivity, PLEs do not present themselves as an alternative to LMS, but as an indispensable complement. PLE can also bring a way to connect and combine individual learning and collective learning. Finally, PLE allows learners to take ownership of their learning and to control their activities. Van Harmelen in [3] proposes a taxonomy of PLEs organized in a multidimensional space: collaborative vs. noncollaborative, closed vs. open, fixed vs. customized, uni vs. multi-institutional, server vs. peer-to-peer or hybrid, onlineonly or mixed. He also mentions three extra-dimensions: a pedagogical approach, a focus on control of the environment and the concept of extensibility and compatibility on many levels. This shows how the problem of integrating PLEs in a learning environment requires a thorough analysis of the 
context of use and of the needs of both learners and institutions that are willing to deploy these solutions for their students.

\section{B. Preliminary Study for the Deployment of PLEs in Higher Education}

The goal of the SWITCH PLE project is to provide technical support and new innovative tools to the federal network of the Swiss universities. In particular, SWITCH mission stresses on bringing new ways to enhance crossinstitutional collaborations and teamwork between Swiss students. In this respect, the collaboration with the Graasp design team aims at seamlessly integrating Graasp in the SWITCH portfolio of educational Internet solutions.

The rationale for the SWITCH network of universities to adapt a PLE platform to their current LMS is multi-fold. First, the idea is to go beyond mere content retrieval and to allow students to contribute within learning activities. Second, in universities, the focus is no longer exclusively on courses but also on teamwork and collaborative learning. As a result, universities need to enrich their LMS ecosystem and provide flexibility both for students and teachers.

In order to better seize the current usage of Information and Communications Technology (ICT) tools by university students, Moccozet et al. conducted a survey among students of the Geneva University [6]. The results of this survey allow setting four main axes for the integration of a PLE within an academic context. A) First, three major types of pedagogical resources have to be considered: 1) local resources, owned and managed by the students on their device(s); 2) institutional resources made available by the university and stored in LMS; 3 ) resources from the cloud, i.e. collected and managed from various Web 2.0 platforms (social bookmarks, social media, Google Docs, etc.). Local and institutional resources remain the most commonly used by students, while cloud resources are still underused because not well integrated in the students' working environment. B) This integration of new tools can be done either directly by the students and/or by the teachers. In any ways, a great care must be taken in finding the right balance regarding the overload for teachers and students implied by the introduction of new tools. C) A rather radical result from Moccozet et al. study [6] is that students are, for a vast majority, not so keen on testing new Web 2.0 tools apart from those already well settled in their habits. D) This finding is, however, counter-weighted in the case of $\mathrm{PhD}$ students who tend to behave as early adopters of new fancy collaborative online tools, due to their need to weave a broad network of intensive collaborations as a necessary boost to their research work.

In the light of their findings regarding current usage of ICT by the Geneva University students, Moccozet et al. established the list of technical requirements and features needed for smooth integration of a PLE within the LMS of the universities that are part of the SWITCH PLE project. These requirements can be summarized as follows. The PLE platform to be used among SWITCH partners should:

- allow the aggregation of local and institutional resources in addition to cloud resources and across the different partner universities catalogs;
- foster collaborative work centered on a user (teamwork) rather than on courses;

- allow to easily extend the system with relevant tools, for instance in a plug and play fashion (as with widgets or browsers' plugins);

- offer a solid and versatile ePortfolio solution. ePortfolio is indeed meant to provide a continuum between formal and informal environments that allow students to manage their content and provide a showcase of their proficiencies and learning outcome while ensuring the interoperability and data mobility from one system to another (e.g., when changing school or institution).

- integrate a dashboard-like feature for better dealing with different tools and platforms.

The PLE solution sought by SWITCH PLE partners should, therefore, facilitate seamless interactions between institutional and non-institutional resources and activities from the students' point of view. Let us now describe Graasp and show how its features meet these requirements.

\section{GRAASP DESCRIPTION IN THE LIGHT OF SWITCH PLE PROJECT REQUIREMENTS}

\section{A. Graasp Overview}

Graasp is a platform that has been developed to investigate the potential of social media in higher education for learning and knowledge management purposes (Figure 1). Graasp contains 4 types of entities: resources, applications, spaces, and people. It targets the management of people's spaces. Graasp supports users in creating and sharing resources and applications with other people in the context of a space. More generally, it is a multi-purpose collaborative platform that assists users in external content and applications aggregation and information organization. In addition, it provides search functionality and guides people with recommendations for fast information retrieval. The privacy control techniques allow users to manage access rights within a space. Applications are currently represented by OpenSocial apps ${ }^{3}$, that bring greater flexibility and extensibility to the system. Let us now explain in more details the notion of a space, which is central to Graasp.

\section{B. Spaces}

Graasp addresses the main requirements to a PLE introduced by Moccozet et al. with spaces [7-9]. A space is an abstract concept that shapes the context in which a user is currently placed. We define a contextual space as an aggregation unit that includes a list of applications that are to be used in the context, a list of people with different access rights sharing the context, resources that can be used in this context, and possibly some other subspaces that belong to this context. A space can represent a group of people, a university course, a company division, a team project, a forum, a blog, etc. It turns out that the space concept can be present in many social environments in one form or another. In Google Groups, Facebook groups, Moodle courses, Dropbox, etc. people meet in a specific context to carry out some activities together. This

\footnotetext{
${ }^{3}$ http://docs.opensocial.org/display/OSD/Specs
} 


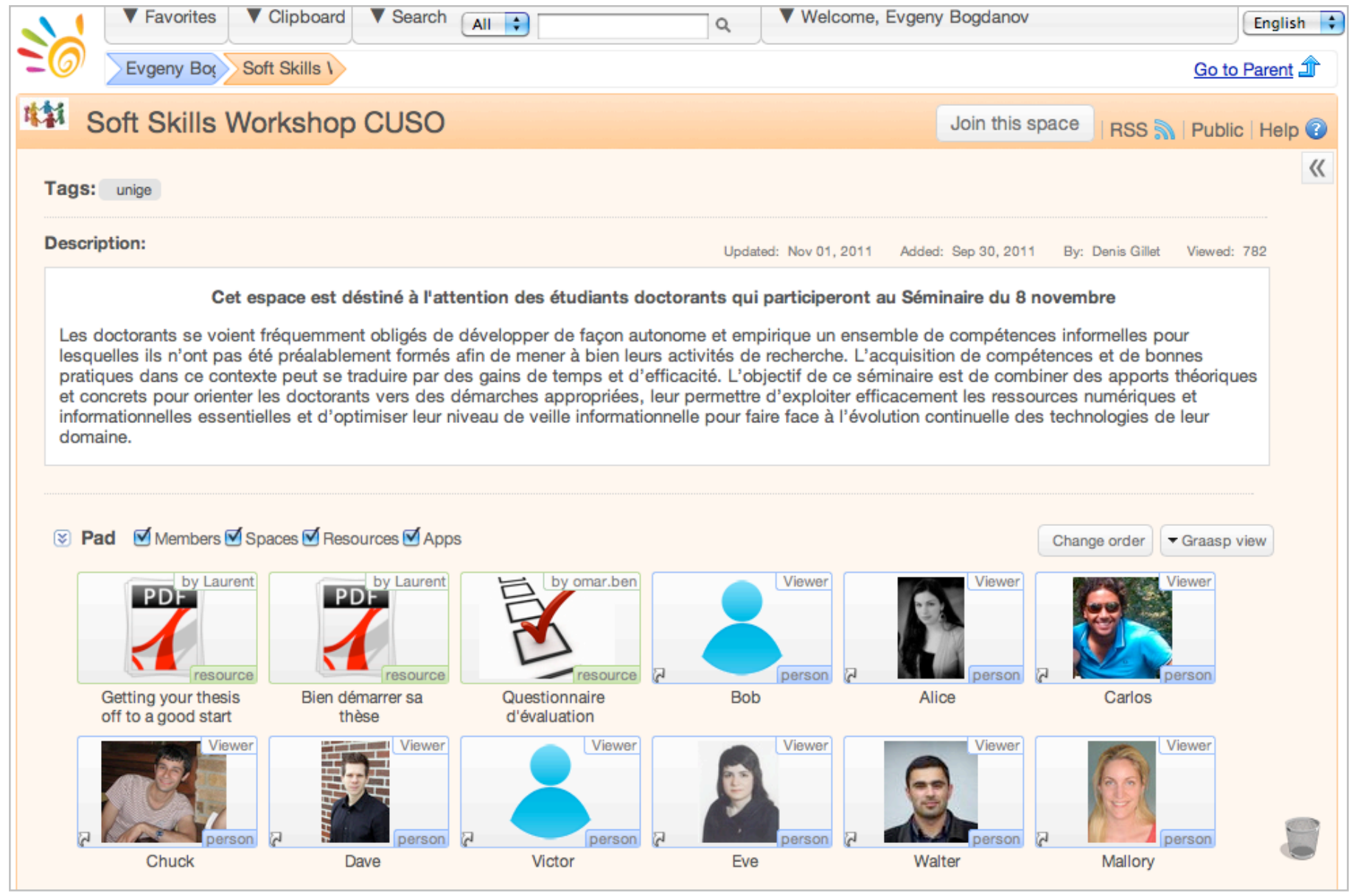

Figure 1. Graasp visual interface

is why the generalization of the context concept (namely, space) was proposed to the OpenSocial specification as a second important concept in addition to people. The main objective of Graasp is to support people in organizing their contextual spaces.

In the light of the SWITCH PLE project requirements, Graasp helps students create and manage their own spaces for teamwork and studying. Through spaces it fosters sharing and collaborative work among students. In addition, it extends the default course-oriented functionality of LMS by providing students with greater flexibility for their study-related resources organization, which shifts a university focus from the pure courses management to the support of user's learning process personalization.

On the other hand, Graasp makes it easier for students to aggregate and organize local, institutional and cloud resources into spaces. The emphasis is made on the simple and efficient accomplishment of these actions. Thus, local resources such as pdf, video, text files can be added into a space by a simple drag-and-drop action from users' desktops. Institutional and external cloud resources such as Youtube videos or Slideshare presentations can be aggregated with GraaspIt! bookmarklet. When a student visits a page of interest, after a click on the GraaspIt! button, the resource is immediately added to Graasp. Later students can rearrange added resources by drag-anddropping them into appropriate spaces.

\section{Learning Oriented Widgets}

Graasp is a highly extensible platform where students or tutors can bring new functionality in the form of OpenSocial apps (one of the standards available for widgets). Thus, Graasp serves as a widget container, where widgets can run and communicate. Space owners can add into their spaces any tool relevant to their learning activities. This capability reinforces the learning experience because it enables useful learningoriented tools to be added and launched during the learning process. Through widgets, one can achieve personalization and adaptation of space behavior to the specifics of learning tasks.

Moreover, different collections of widgets can be associated to different spaces, making the aggregation contextual. Widgets can be bundled together with other resources in spaces and, thus, be directly linked with relevant content. This feature alone allows teachers to provide handy learning kits that learners can further customize and share. Thanks to this feature, Graasp's provided functionalities are made flexible and extensible. For instance, in a project space, students can add a calendar widget configured with a series of milestones and deadlines. They can easily add simulation widgets, educational games or lightweight remote laboratory clients as well as many domain-specific widgets. 
In the context of the ROLE EU-funded project, a widget store was implemented that provides browsing capabilities to search for widgets aimed for learning purposes ${ }^{4}$. The widget store has already a learning-oriented widgets pool and the number of available widgets is constantly growing. Graasp provides simple ways to aggregate widgets from the widget store. In case the user is browsing through widgets in the store, a widget of interest can be added into Graasp through a click on the GraaspIt! bookmarklet. The functionality of viewing widgets available in the store directly inside Graasp (when managing a space) is supported.

\section{Privacy Management}

Since Graasp provides a relatively open learning environment, there is a clear need for effective privacy control mechanisms that protect against unauthorized access to social data. Instead of adopting complicated privacy management schemes that are difficult for users to cope with, the privacy settings are maintained at the space level.

Based on its purpose and its owner's choice, a space can be public, closed, or hidden. Public spaces are globally visible and allow every user to join. Closed and hidden ones are only accessible upon explicit invitations. Hidden spaces are not searchable and they are only visible to space members. Closed and hidden spaces are especially useful when students want to carry out their peer-based projects without being disturbed by others or feeling that they are "observed" by the tutor.

Within a specific space, users are allowed to take different roles: owner, editor, and viewer. Each role is associated with a set of rights allowing users to perform diverse actions such as moderating the space, adding new assets in the space, commenting, rating, tagging, bookmarking, etc. Assigning different roles in a collaborative space makes users aware of their duties and gives them the opportunity to concretely collaborate by being allowed to perform specific actions.

\section{E. Extensions for Managing Competencies}

The current trend of massive individualization of training has inherently several aspects. On the one hand, students with different backgrounds may now, for instance, more often follow the same master program or later occupy the same position. On the other hand, and more globally, the faster pace of technology evolution implies a greater need in just-in time and just on demand training. To tackle these new challenges in the context of collaborative learning, we propose inviting learners to develop their competences in a more agile way by taking part in a currency-free bartering scheme of coaching and learning services given in exchange of credit units. This scheme takes up the form of an extension to Graasp that we named Competence Bartering Platform (CBP).

With the CBP, learners who turn temporarily into teachers (or coaches) get official recognition of their skills in the form of credit units. These credit units can later be invested to get new competences from other peers. The multilateral exchange principle allowed by the use of credit units overcome the implicit bilateral exchange scheme whereby A helps B who helps $\mathrm{A}$ in return. The CBP is developed as a part of the ROLE

\footnotetext{
${ }^{4}$ http://www.role-widgetstore.eu
}

project and in close relation to the objective to foster selfdirected learning. It also fits in the requirements of the SWITCH PLE project to provide an ePortfolio solution to their students, in particular regarding the management and development of their own competences. As students enrich their competence profile, they become more visible to other peers seeking for missing competences, and so on. The inherent cold-start effect is easily overcome by offering free credit to new students and by setting a negative interest rate as advised by Liaeter [10].

Technically, this platform comes as an extension to Graasp built-in services plus a set of dedicated widgets that allow users to 1) provide and maintain their competence profile, so that these data become searchable and recommendable by Graasp built-in functions; 2) communicate with potential trading partners; and 3) manage payment and billing with trading partners upon completion of the informal teaching sessions. Let us now detail how these functionalities are provided by the CBP.

The CV Builder widget allows users to edit and manage their CV with the possibility at any time to export a Europass 5 compatible pdf version (Figure 2). It can be seen as a nonintrusive way to collect competence data while still providing a useful service to users. It makes use of the Europass Web services to generate the pdf, which embeds also xml CV data. The downloaded file is, thus, compatible with other third party services that follow Europass standards. The description of skills and competences in this tool are based on the European Qualification Framework (EQF) that proposed an 8 level scale to describe the level in a competence ${ }^{6}$. A competence is, thus, modeled as a combination of a concept denoting a scope or a topic and an indication of the level in the EQF scale.

All the CV information provided is stored within Graasp, skills and competence data are thus available to the search and recommendation built-in services. Since OpenSocial standards do not yet support competences as a part of a user's profile, we proposed an extension to the OpenSocial user profile data specification described in detail in [11] and implemented in Graasp. One advantage of including competence management within a space-enabled social media platform such as Graasp is that it enables agile recommendation of competences relevant to the context. For example, each time students browse their spaces, they are recommended people with competences relevant to the topic of each space.

Once a relevant person with a sought-for competence is found, a bundle of already existing communication tools can be used to arrange and conduct coaching sessions. The ROLE Widget Store provides a series of such tools that can run in Graasp $^{7}$ (chat, calendar, or video conference such as FlashMeeting). Providers of coaching sessions can ask in return "Educational Credit Unit" (ECU) using the Credit and Trades management widget. This widget, currently in a development stage, allows issuing a bill, paying a trading

\footnotetext{
${ }^{5}$ http://europass.cedefop.europa.eu

${ }^{6} \mathrm{http}: / /$ www.emcq.eu/index.php?page=the-eqf-reference-levels

${ }^{7}$ http://www.role-

widgetstore.eu/tools/Collaborate $\% 20 \% 2526 \% 20$ Communicate
} 
partner, and checking one's credit balance. This tool relies on the Credit Management service that safely stores users' credit accounts and allows them to secure their transactions. This service is based on Rivulet ${ }^{8}$, a back-end implementation of the Ripple-project monetary model ${ }^{9}$. This monetary model was thought of as a way to overcome the community currencies (typically used in LETS ${ }^{10}$ schemes) whereby "consumers" of a service are granted credit by the community as a whole without any guaranty that "consumers" will give back in return at some point. In Ripple model, credits are granted directly between two people who trust each other, or through a path within the trust network weaved between community members. Thus, this model fits particularly well in the context of learners' networks that rarely form solid and durable communities needed for a classical community currency to work (for a more detailed explanation on the choice of this model, see [11]).

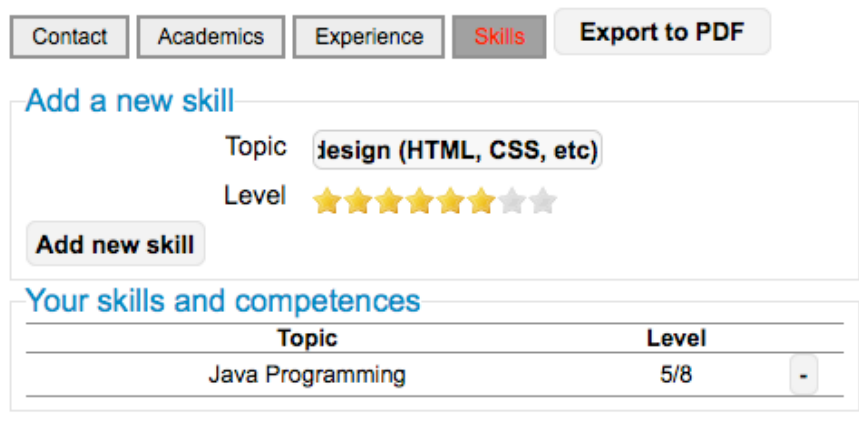

Figure 2. CV Builder widget screenshot (focus on "skills" tab)

\section{F. Cross-recommendation Service Design}

To take into account the specific requirements of the SWITCH PLE project, we are currently designing a cross-recommendation service to allow for recommending resources from different provenance. Graasp, in its current implementation, features a recommender system that analyzes the resources, all data related to the applications, all the members of the platform and suggest relevant resources, applications, or people at any point of the navigation process. This service explores the usage of learning widgets, shared resources, activity spaces, and the interactions between learners and experts in different learning contexts (see [12] for a more detailed description of the recommender). The main idea for the cross-recommendation service is to implement a recommender engine to collect and explore the information from the different institutions. After the recommender engine is set up, it will send requests to these institutions periodically. As on each platform it will record users' behavior and the corresponding resources in a unified format. Then, it will return to the recommender engine the result of the requests in a specific format. With the result, the recommender engine can explore the data and set up a graph that indicates the relationship among users and resources, then the 3A Ranking

\footnotetext{
${ }^{8} \mathrm{https} / / /$ github.com/jplewicke/rivulet

${ }^{9} \mathrm{http}: / /$ ripple-project.org

${ }^{10}$ LETS were created in the 80 's as an attempt to promote mutual support communities and alternative forms of trade. In these communities, members can trade skills in the form of services in a multilateral way and in exchange of credit units.
}

Algorithm [12] will be applied to it and can make the recommendation to the target user in specific contexts.

Thanks to the data aggregated from several institutions, the recommendation service will then be able to deliver both internal and external recommendations based on the target users' context, triggering by that learning and interaction opportunities beyond institutional boundaries. Knowing that the universities involved in the project all rely on Shibboleth (for Single Sign On), Graasp is being adapted to offer a logging mechanism using this standard. In addition, a scheme for exchanging interaction data with the recommendation engine(s) is explored. To encourage the sharing and expansion of knowledge objects whilst respecting copyright and privacy issues, access to every recommended Web item will be verified via Shibboleth.

\section{Evaluations}

We have already tested Graasp, as a tool meant to help students in their collaborative work or personal resources management, in two different real-world experiments, and we have already deployed a dedicated instance of Graasp for the SWITCH PLE project where it is currently used by the students. The following sub-sections go into more details for each of these evaluation campaign, and in Figure 3 we give the quantitative results we obtained from the usage logs for the first two evaluations.

\section{A. Tongji University Evaluation}

To examine the acceptability of Graasp in terms of supporting collaborative learning, it was used as a collaborative work platform in a project-based course of "Human Computer Interaction" offered at Tongji University in China. 28 undergraduate students were involved in the course, and they were divided into 8 teams. Each team was asked to accomplish a group project, and Graasp was introduced to the students at the beginning of the course. Students were entitled to create their project spaces, share resources with each other, play different roles in the project, and work with different learningoriented apps. A survey was conducted with the students participating in the course, aiming at evaluating Graasp's acceptability in sustaining collaborative learning. The quantitative results from the usage logs are shown in Figure 3 for comparison with the Geneva experiment, and the (qualitative) results of the survey have been published in [13]. Let us now summarize the main outcome of this experiment.

Evaluation results show, that in general Tongji students who took part in this experiment were satisfied with using Graasp to enhance knowledge management and collaboration while preserving the privacy of their social data. Most students expressed their preference for carrying out teamwork within project spaces and confirmed the usefulness of integrating apps into their learning process. In addition, students considered Graasp to be convenient in organizing content with subspaces and tags, but these features were less exploited due to the lack of resources existing in the platform. Finally, participants felt it necessary and helpful to have control over the privacy levels of spaces and user profiles. 


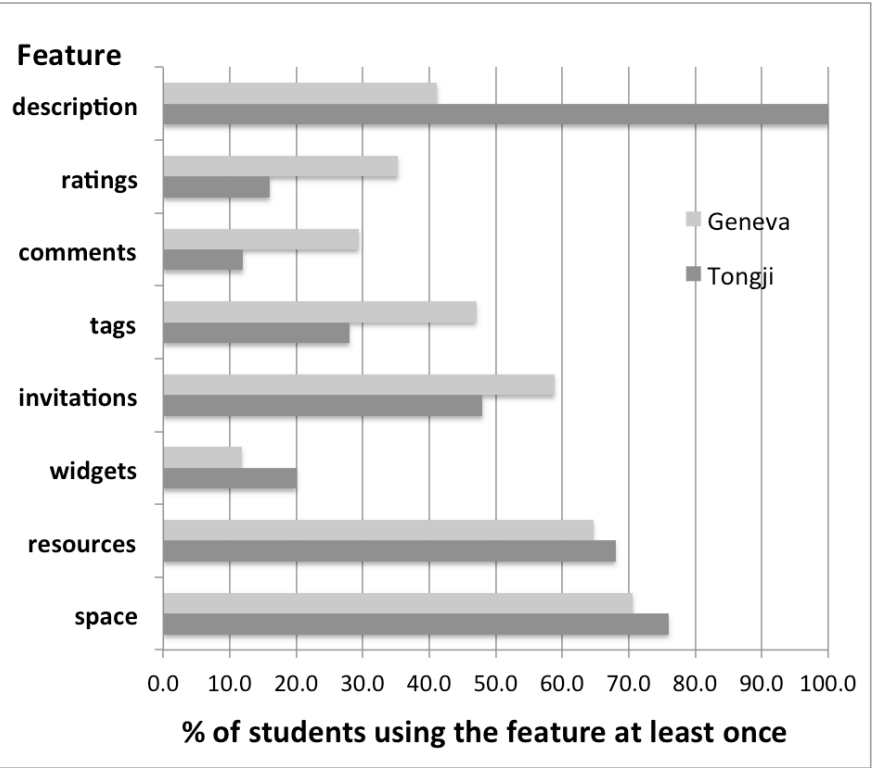

(a)

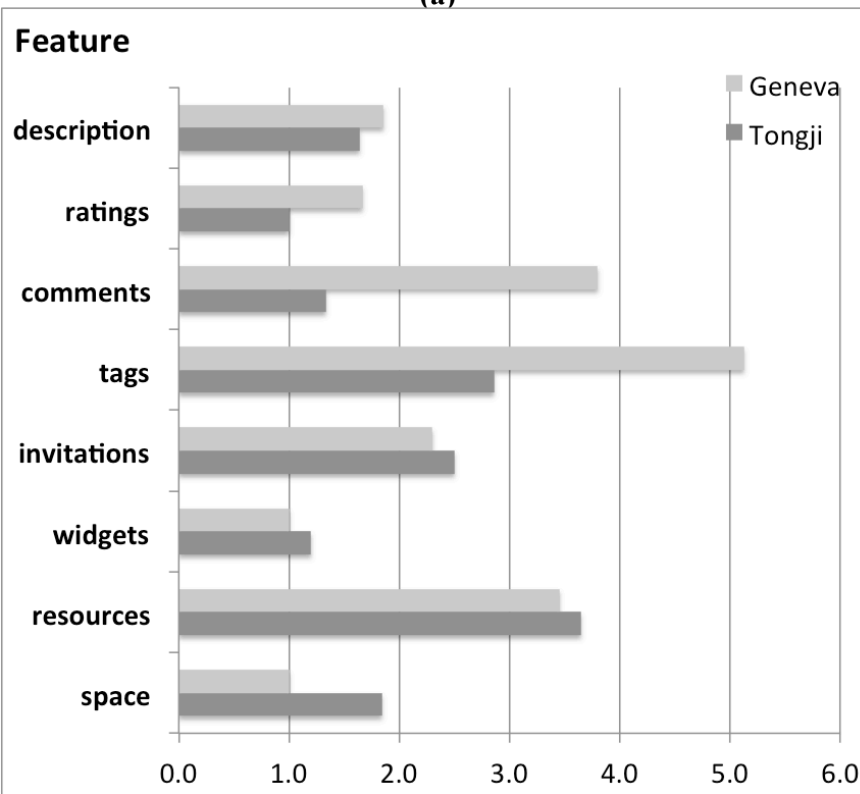

Average number of items created per student

(b)

Figure 3. Quantitative measure extracted from the logs of different Graasp's features usage during evaluation experiments at Geneva workshop and Tongji University. The figure (a) shows the percentage of students who used a feature at least once (excluding the auto-created personal home space), and the figure (b) shows the average number of items created per student (excluding the students who did not use the corresponding feature). The features are referred to as the following: for any resource in Graasp, users can link a textual description, a "star-based" rating, a comment, or some tags; users can also create spaces, and link some resources or widgets (applications) to them and send space invitations to anyone.

\section{B. Geneva Softskill Workshop Evaluation}

The second real-world experiment was held at the occasion of a workshop, entitled Softskill, organized by the Geneva University and targeted to new PhD students. The aim of this workshop was to give some advice regarding research work in general, and introduce them with most useful Web 2.0 tools, hence the name Softskill. For the organizations, the tutors of this workshop extensively used Graasp to communicate, to collect, and to organize in different sub-spaces the material for the workshop (N.B. the usage data for the tutors is not included in data shown in Figure 3). Students who had registered to the workshop (17 in total) have also been invited almost 2 weeks in advance and, thus, got a chance to get accustomed with the tool, before the actual session, by registering and creating their own personal profile (N.B. this usage data has been discarded in Figure 3). During the workshop, the students, after a short introduction, were handed a small exercise $(30 \mathrm{~min})$ to perform using Graasp. In the scenario for this exercise, students were about to write a survey paper and had to collect some relevant material resources, organize them with spaces and/or tags (according to their own convenience), and then share them (by sending invitations) with other people for comments or review.

The quantitative metrics that we extracted from usage logs are shown in Figure 3 (bars in light grey for the Geneva workshop). The results regarding the use of spaces, resources, widgets, and invitations are in line with those obtained from Tongji students, with a number of items created per student remaining lower for Geneva (especially for spaces), that can be explained by the shorter time frame of the latter experiment. However, PhD students from Geneva seem clearly more confortable and keen on using tags, comments, and ratings, i.e. typical web 2.0 and collaborative features. Besides the fact that in the Geneva experiment students were explicitly invited to use tags (but not forced to), unlike the Tongji experiment, the usage ratio and average number of items created by students is higher in the Geneva experiment. This result is even reinforced by the shorter time frame of the Geneva experiment and overall confirms the survey of Moccozet et al. that showed that $\mathrm{PhD}$ students were more enthusiasts than undergraduates (as in the Tongji experiment) on the use of collaborative Web 2.0 tools.

The qualitative results in Figure 4 show the perceived usefulness of Graasp for aggregating, organizing, and sharing of resources. These results are taken from a quick and short questionnaire answered at the end of the exercise by 13 out of the 17 registered students. One thing to notice is that, beyond the usefulness of Graasp as a whole to aggregate and share resources, the majority of users found the space notion useful for organizing resources. This is a significant result that shows the added value brought by Graasp since the space notion was unknown to most of the students. These results show more generally that the majority of students who answered the questionnaire agree that Graasp is useful for those three purposes and, as such, suited both for personal and collaborative work.

\section{Graasp Deployment for SWITCH PLE}

The big scale evaluation of Graasp is currently undergoing within the SWITCH PLE project. The project plan is to introduce Graasp as a complementary technology to the existing in universities LMSs, where Graasp serves as an aggregation dashboard that makes a bridge between university courses-oriented technologies from one side and Personal Learning Environments from another side. The goal of using Graasp is to help students aggregate and organize into spaces 
local, institutional and cloud resources as well as share them with other people.

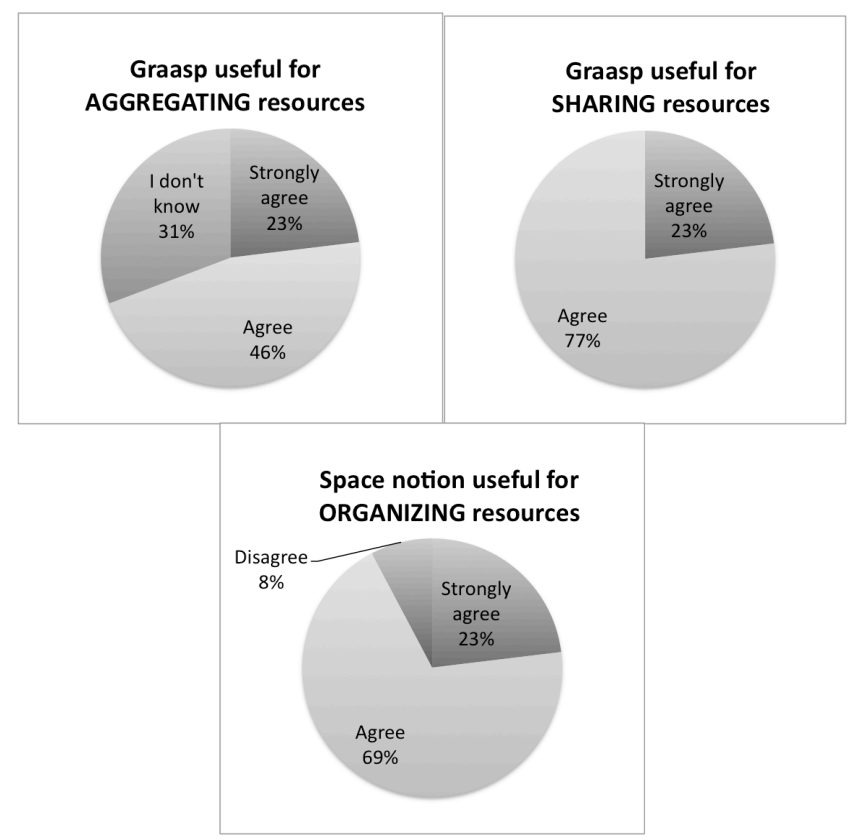

Figure 4. Qualitative results for the Geneva Experiment concerning Graasp's usefulness for aggregating, organizing, and sharing of resources.

To integrate Graasp within university, we provided a local installation of the platform to each university. Graasp's visual appearance is adapted to university branding requirements and Shibboleth authentication is integrated inside to provide an easy Single Sign On mechanism to university students. The integration with existing university LMS (Moodle, Mahara, Mediaserver, etc.) is two-fold. On the one hand, a resource from a LMS can be aggregated into Graasp with GraaspIt! plugin. On the other hand, the cross-recommendation technique will provide recommendations within Graasp, where recommended items will be harvested from both Graasp and LMSs resources as well as resources from other universities.

Graasp is now being incrementally introduced to the students of Geneva and Fribourg Universities starting with small groups of master and $\mathrm{PhD}$ students and later engaging the whole students population counting to 30000 people. This evaluation campaign is spanning over a longer period finishing in the beginning of 2013, so final results are not yet available. However, it has already been demonstrated that Graasp, as a flexible social media platform, can be easily integrated in various institutional settings, optionally as a complement to an existing LMS. Its smooth introduction in curricula to bring added value in supporting teamwork and improving personalization of learning process is an interesting scheme to ease adoption and trigger a paradigm change in the institutional IT landscape. In particular, the possibility to fulfill diverse needs by extending traditional LMSs with learning widgets, suggested by teachers, but personalized and enriched by students, is highly valued.

\section{CONCLUSION}

This paper discussed the usage in a learning context of Graasp, a social media platform that simultaneously offers a bottom-up project management platform, a resource repository, a collaboration site, and a widget container. This platform, albeit suited for any field of application, has proved to be useful to support students in scientific disciplines while conducting collaborative projects.

The evaluation of its acceptability and usefulness has been conducted among undergraduates in Tongji University and $\mathrm{PhD}$ students from Geneva University. Both experiments showed that students were satisfied with using Graasp to collect, organize, and share resources with their peers. More specifically, the space notion, which was quite new to most of them, seemed natural to all students and helped them to enhance knowledge management and collaboration. Another longer-term study, involving courses that span over a longer period of time, is also being conducted within the SWITCH PLE project. The goal of this latter ongoing study is to verify and further evaluate the usefulness of Graasp for the specificity of collaborative learning, agile content aggregation and sharing, and, more generally, full learning project management.

With Graasp, current LMSs are complemented by administrator-free spaces creation and learning-oriented widgets. These features extend the LMS functionalities by adding more flexibility in learning process organization and personalization to both students and tutors. With this, we hope to trigger a paradigm shift in universities from courses management to the personal development of each learner and enhancement of students' teamwork. Graasp also features an extension for competence management and development, the Competence Bartering Platform, which is highly relevant to the ePortfolio program in the SWITCH PLE project. This module offers competence management through the CVBuilder widget and Graasp's built-in search and recommendation engine in order to provide contextualized opportunities to learners to gain new competences from their fellow learners. This approach also brings in new perspectives for more agile development of competences and a new framework we can call cloud coaching.

If we now go back to the four main axis proposed by Moccozet et al. regarding the integration of a PLE within an academic context, we see that Graasp offers flexible and concrete solutions to suit this purpose. First, Graasp allows students (and teachers) to gather and organize local, institutional, and cloud resources either through bookmarking online resources or uploading local files and linking them to as many spaces or sub-spaces they wish. Second, Graasp can be used indifferently by students or teachers, while fully preserving the privacy for both parties and allowing transparent collaboration and sharing through invitations and setting of access rights. Indeed, space owners can invite whomever they want and make the content of a space visible and/or modifiable for a precisely selected group of people. Third and fourth in Moccozet et al. analysis, Graasp has proven to be useful both for undergraduate and $\mathrm{PhD}$ students. Moreover, as an open yet privacy-aware social platform, users can quickly evolve from mere lurkers, reading content posted by other users, to power users dealing with private-only work or restricted access 
collaborative projects through multiple spaces. Unlike typical social networks platform, which require users to weave a substantial web of contacts before getting the full benefit of the tool, Graasp connects people through their concrete activity and always within relevant contexts (via the space notion), thus, allowing a greater variety of usage.

Our future plans are to explore Graasp use by students and further improve and adapt it to their learning activities as well as to conduct big scale usefulness and usability evaluations with students.

\section{ACKNOWLEDGMENT}

The research work described in this paper is partially funded through the ROLE Integrated Project; part of the Seventh Framework Programme for Research and Technological Development (FP7) of the European Union in Information and Communication Technologies, and the Personal Learning Environment (Phase 3) project of the AAA/SWITCH programme.

\section{REFERENCES}

[1] N. Dabbagh and A. Kitsantas, "Personal learning environments, social media, and self-regulated learning: A natural formula for connecting formal and informal learning," Internet and Higher Education (2011), in press.

[2] N. Li, C. Ullrich, S. El Helou, and D. Gillet, "Using social software for teamwork and collaborative project management in higher education," Proc. International Conference on Web-based Learning, vol. 6483, pp. 161-170

[3] M. Van Harmelen, Personal Learning Environments, Sixth IEEE International Conference on Advanced Learning Technologies (ICALT'06), 815-816, 2006

[4] C. Severance, J. Hardin, and A. Whyte. The coming functionality mashup in Personal Learning Environments. Interactive Learning Environments, 16(1):47-62, 2008.

[5] F. Henri, B. Charlier, and F. Limpens, Understanding PLE as an Essential Component of the Learning Process. ED-Media AACE, 2008

[6] L. Moccozet, O. Benkacem, B. Ndiaye, V. Ahmeti, P. Roth, and P.-Y. Burgi. An exploratory study for the implementation of a technopedagogical personal learning environment. Proceedings of the The PLE Conference 2011, 2011.

[7] E. Bogdanov, C. Salzmann, and D. Gillet. Contextual Spaces with Functional Skins as OpenSocial Extension. In 4th International Conference on Advances in Computer-Human Interactions, 2011.

[8] S. Sire, E. Bogdanov, M. Palmér, and D. Gillet. Towards Collaborative Portable Web Spaces. In 4th European Conference on Technology Enhanced Learning (EC-TEL) - Workshop on Mash-Up Personal Learning Environments (MUPPLE-09), 2009.

[9] S. El Helou, N. Li, and D. Gillet, The 3A interaction model: towards bridging the gap between formal and informal learning. Proceedings of The Third International Conferences on Advances in Computer-Human Interactions. (2010)

[10] B. Lietaer, Community Currencies: A New Tool for the 21st Century, http://www.transaction.net/money/cc/cc01.html, 2001

[11] F. Limpens and D. Gillet, Currency-free exchange platform, deliverable D4.4 Responsive Open Learning Environments - European Commission Seventh Framework Project (IST-231396), 2011

[12] S. El Helou, C. Salzmann, and D. Gillet, The 3A Personalized, Contextual and Relation-based Recommender System Journal of Universal Computer Science, 2010, 16, 2179-2195

[13] N. Li, S. El Helou, and D. Gillet. Using social media for collaborative learning in higher education: A case study. In Proceedings of 5th International Conference on Advances in Computer-Human Interactions, 2012, to appear
[14] G. Boyd, Editorial: Quatts, virtual currency for gaming and bartering education on the Web, British Journal of Educational Technology, 2002, 33, 361-363

[15] R. Fugger, Money as IOUs in Social Trust Networks \& A Proposal for a Decentralized Currency Network Protocol, http://rippleproject.org/decentralizedcurrency.pdf, 2004

[16] D. Gillet, S. El Helou, C.M. Yu, and Ch. Salzmann, "Turning Web 2.0 Social Software into Versatile Collaborative Learning Solutions", The First International Conference on Advances in Computer-Human Interaction (ACHI'08), Sainte Luce, Martinique, February 10-15, 2008.

[17] W. Reinhardt, C. Mletzko, H. Drachsler, and P. Sloep. AWESOME: A widget-based dashboard for awareness-support in Research Networks. In Learning, num- ber 1. PLE Conference;, 2011.

[18] J. L. Santos, K. Verbert, S. Govaerts, and E. Duval. Visualizing PLE Usage. In Proceedings of EFEPLE11 1st Workshop on Exploring the Fitness and Evolvability of Personal Learning Environments. CEUR workshop proceedings, 2011. 